\title{
Color Enhancement Strategies for 3D Printing of X-ray Computed Tomography Bone Data for Advanced Anatomy Teaching Models
}

\author{
Megumi Inoue ${ }^{1,2}$, Tristan Freel ${ }^{2}$, Anthony Van Avermaete ${ }^{2}$ and W. Matthew Leevy ${ }^{1,2,3, *}$ \\ 1 Department of Biological Sciences, 100 Galvin Life Science Center, University of Notre Dame, \\ Notre Dame, IN 46556, USA; minoue1@nd.edu \\ 2 IDEA Center Innovation Lab, 1400 E Angela Blvd, South Bend, IN 46617, USA; \\ school.tristan.freel@gmail.com (T.F.); avanaver@nd.edu (A.V.A.) \\ 3 Harper Cancer Research Institute, University of Notre Dame, Notre Dame, IN 46556, USA \\ * Correspondence: W.M.Leevy.2@nd.edu; Tel.: +57-4631-1683
}

Received: 22 January 2020; Accepted: 17 February 2020; Published: 25 February 2020

\begin{abstract}
Three-dimensional (3D) printed anatomical models are valuable visual aids that are widely used in clinical and academic settings to teach complex anatomy. Procedures for converting human biomedical image datasets, like X-ray computed tomography (CT), to prinTable 3D files were explored, allowing easy reproduction of highly accurate models; however, these largely remain monochrome. While multi-color 3D printing is available in two accessible modalities (binder-jetting and poly-jet/multi-jet systems), studies embracing the viability of these technologies in the production of anatomical teaching models are relatively sparse, especially for sub-structures within a segmentation of homogeneous tissue density. Here, we outline a strategy to manually highlight anatomical subregions of a given structure and multi-color 3D print the resultant models in a cost-effective manner. Readily available high-resolution 3D reconstructed models are accessible to the public in online libraries. From these databases, four representative files (of a femur, lumbar vertebra, scapula, and innominate bone) were selected and digitally color enhanced with one of two strategies (painting or splitting) guided by Feneis and Dauber's Pocket Atlas of Human Anatomy. Resulting models were created via 3D printing with binder-jet and/or poly-jet machines with important features, such as muscle origin and insertion points, highlighted using multiple colors. The resulting multi-color, physical models are promising teaching tools that will enhance the anatomical learning experience.
\end{abstract}

Keywords: 3D printing; anatomy education; multi-color printing

\section{Introduction}

Three-dimensional (3D) printing revolutionized the production of accurate anatomical models in a timely and cost-effective manner. Biomedical data sourced from computed tomography (CT) or magnetic resonance imaging (MRI) scans are typically used as the blueprints for the production of patient-specific and/or educational physical models. While the latter were already introduced into anatomy classrooms, they largely remain monochrome. For example, Smith et al. evaluated the use of various single-color 3D-printed anatomical models of the respiratory and musculoskeletal system in an undergraduate laboratory [1], while Abou Hashem et al. successfully integrated analogous bone models in the classroom [2]. Previously, we reported the creation of models for patient education in a clinical setting [3]. Since then, advancements in 3D printing technologies now allow for the creation of rapid, full-color prototypes. Multi-color, physical 3D models have great potential to enhance the anatomical learning experience for students and clinicians alike, with one report using the upper limb in the education of medical students [4]. 
Anatomy is an inherently 3D discipline that cannot be fully expressed in the two-dimensional (2D) world, whether in textbooks or in the virtual space. Physical 3D models possess several advantages over virtual and illustrated models as they allow learners to "see with their hands" [5]. This tangibility translates to a strong preference for 3D models among students and researchers alike [6]. Khot et al. concluded that physical models are more effective in student learning than computer-based modalities such as Key View (photographed views of a plastic model in a PowerPoint presentation) and virtual reality (3D color image reconstruction from CT) for the study of pelvic anatomy [7]. Preece et al. came to the same conclusion confirming the visuospatial advantages of physical models over textbooks and 3D computer models for veterinary students learning equine foot anatomy [8]. Garas et al. affirmed the feasibility of 3D-printed models as a valuable asset in anatomy learning and showed their capability to be used adjacent to cadaveric materials and other widely used tools in anatomy education [9]. In this study, we demonstrate and compare two pathways for the creation of advanced anatomical models suitable for medical education by interfacing biomedical data with multi-color 3D printing instruments.

There are two conventional options for generating a multi-color, 3D-printed part: (1) poly-jet printing, which uses ultraviolet (UV) photo-cured resin or wax, and (2) binder-jet printing, which uses powder and a binding agent. While other options include the recently released Da Vinci Printer (XYZ Printing Co.) that mixes filaments, or manually printing each part in a separate color with subsequent assembly, the poly-jet and binder-jet remain the most commonly accessible multi-color options. In poly-jet printing, liquid photopolymer resins are jetted through printhead nozzles as droplets before being cured by a UV light [10]. Systems with multiple nozzles that jet different colors enable multi-color printing since liquid materials may be mixed in a given volume before curing. Poly-jet printing with the Stratasys Objet Connex3 series printers is well documented in the production of a wide range of anatomical models that serve as both pre-operational and educational aids. Pioneers of poly-jet printing in medicine produced color-coded heart models [11], upper limb vasculature with a colored distinction between nerves, arteries, veins, and musculature for medical students [4], and personalized kidney models showing tumor and neurovasculature for patient education [12]. Multi-material printed models also successfully assisted surgical planning for a septal myectomy [13] and tumor resections in the left and right atria [14]. Models utilizing clear polymers proved especially beneficial as curricular aids. Five common ventricular septal defect (VSD) subtypes obtained from a radiology archive were printed with poly-jet in a Stratasys Objet Connex printer to supplement the education of 23 pediatric residents at the Children's National Health System in Washington, DC [15]. These residents reported superior knowledge acquisition, enhanced ability to recall knowledge, and proficiency in conceptualizing the structure of VSDs.

Binder-jet printing produces models by using a binding agent that fixes a powder-based substrate [16]. This method permits the use of many kinds of materials including metals, polymers, and ceramics. In particular, full-color sandstone printing, in which an inkjet printhead can apply color to each printed layer, has been well utilized commercially and in anatomical teaching. Manufacturers use this technique to print photo-realistic, full-color prints of architectural models, lifelike sculptures, memorabilia, and complex models [17]. In anatomy, sandstone binder-jetting was used to replicate specimens like a portion of a fossilized hydrosaur femur [6] from overlapped photography and a full-size colored replica of a cadaveric upper limb [18] from CT. Cadaveric reproduction with binder jetting in full color also facilitated the creation of a human head, neck, and orbital model with data collected from a 3D scanner to provide a haptic experience for training ophthalmologists [19]. Additionally, it was used to create a color-coded model depicting the anuran outflow tract of a cane toad heart to demonstrate the use of full-color printing in reproducing complex anatomy [6].

Traditionally, researchers achieve color assignment for a 3D print by separating a dataset into multiple files through segmentation of different voxel values (in units of density or intensity). This process historically restricted printing to heterogeneous tissues with significant density differences to enable separate pieces to be produced with corresponding colors. This process was used to produce multi-material models that showcase the collecting system within a kidney [20], tumor and liver 
vasculature [21], and bone with soft tissue in the ear, nose, and throat [3]. Color-coded patient specific heart models were utilized in surgical planning [11], in which the density difference between blood and surrounding cardiac tissue was used by an automatic segmentation algorithm to create a binary map for 3D reconstruction. In some cases, the density differences may be accessed via multimodal CT/MRI imaging datasets to create a multi-color print of soft and hard tissue. MRI data were used to create surgical models for trans-nasal endoscopic surgical training and multi-color printed with different colors for the skull, nerves, arteries, and veins [22]. While segmentation is a powerful tool, it functionally struggles within tissues of homogeneous density. As such, alternate approaches are necessary, especially if sub-structure labels are desired outputs.

In this study, we successfully generated multi-color anatomical models that are difficult to create with traditional segmentation strategies. Examples deploying this methodology described herein are exceedingly rare, especially in the specific case of manually highlighting important features of bone such as muscle origin and insertion points, on a model derived from biomedical data. While cadaveric reproductions were created using similar methods, our color models enhance the learning experience by adding information to the 3D models. We utilized high-resolution, 3D reconstructed, monochrome surface models derived from open access libraries as the starting point. Four bone models were selected of a femur, lumbar vertebra, scapula, and innominate bone. Digital color enhancement was demonstrated with two separate strategies to detail pathways that leverage poly-jet or binder-jet printing modalities. The Pocket Atlas of Human Anatomy, by Feneis and Dauber [23], was used to guide the digital painting process.

Resulting models were created via 3D printing with binder-jet and/or poly-jet machines, with important features such as muscle origin and insertion points highlighted using multiple colors. These strategies will enable students, educators, and researchers to create a wider array of multi-color, physical models true to biomedical data to enhance the anatomical learning experience.

\section{Materials and Methods}

\subsection{Data Processing}

\subsubsection{For Poly-Jet Split Processes}

The 3D files of a femur (NIH 3D Print Exchange, Model ID: 3DPX-000168) and lumbar vertebra (NIH 3D Print Exchange, Model ID: 3DPX-000899) were obtained as stereolithography (STL) files through the NIH 3D File Exchange website. The files were then imported into Meshmixer (Version 3.5.474), which is a modeling software freely available for download. Features were selected and separated as multiple STL files using three tools: "Create Facegroups," "Generate Complex," and "Split Complex." While other 3D modeling software allows for splitting a model with a plane, the "Generate Complex" tool in Meshmixer allows for organic selection and splitting according to the shape of the model. Selection of important regions was done by referencing the Pocket Atlas of Human Anatomy. The generated files were exported as separate STLs with the selection "Export as STL Binary Sequence-One File per Region."

\subsubsection{For Binder-Jet Painting Processes}

The 3D files of a femur (NIH 3D Print Exchange, Model ID: 3DPX - 000168), lumbar vertebra (NIH 3D Print Exchange, Model ID: 3DPX-000899), scapula (GrabCAD, Scapula-Human Anatomy, uploaded by Hadi Nasution), and innominate bone (Sketchfab, Os-Coxae, uploaded by kuehn) were obtained through respective online libraries in STL format and imported into Meshmixer. The surfaces of these models were painted using the PaintVertex tool with a mouse and a Wacom Intuos Pro graphics tablet guided by the Pocket Atlas of Human Anatomy [23]. Utilizing the graphics tablet allowed for easier coloring and higher resolution selection. The colored models were exported as VRML (Virtual Reality 
Modeling Language) files and printed in sandstone using third-party 3D printing service Shapeways. We uploaded our colored bone models online, which we uploaded for public access on Sketchfab.

- Femur: https://skfb.ly/6Mt8w;

- Lumbar: https://skfb.ly/6MYTw;

- Scapula: https://skfb.ly/6MYTL;

- Innominate bone: https://skfb.ly/6NotO.

\subsection{D Printing}

\subsubsection{For Poly-Jet Processes}

Split STLs were subsequently imported into 3D printing software GrabCAD Print ("File" > "Add STLs as an assembly.") GrabCAD Print allows for a palette of mixtures among selected materials. For the Connex3 Objet350 used here, three materials in VeroClear, VeroCyan, and VeroYellow were loaded for mixing. Visually distinct colors from this palette were selected for each part as a different material and assigned to each part.

\subsubsection{For Binder-Jet Processes}

VRML files were directly uploaded onto the Shapeways (shapeways.com) website and resized for handling and price considerations. Within the Shapeways website, under materials, "full-color sandstone" with a "natural finish" was chosen.

\section{Results}

\subsection{For Poly-Jet Split Processes}

Anatomical datasets of the femur and lumbar spine were selected from the NIH 3D Print Exchange website. The "Split Complex" tool in Meshmixer was utilized to cut the two files into separate subdomains using the Pocket Atlas of Human Anatomy by Feneis and Dauber as an anatomical reference guide. The components were assigned color and reassembled in GrabCAD software prior to printing on a Connex3 Objet350. This machine was capable of using three "mixable" materials and was loaded with VeroClear, VeroCyan, and VeroYellow such that six colors could be generated and distinguished by eye: blue, yellow, green, clear, light green, and light blue.

The original 3D model and its "split" parts of a left femur are shown in Figure 1, Frame A, as a digital scene split in Meshmixer and recompiled in Meshlab. The final physical model is shown in Frame B. The femur shown in Figure 1 highlights the following features: 1. head, 2. lesser trochanter, 3. intertrochanteric line, 4 . body, 5 . medial condyle, 6 . lateral condyle, 7 . gluteal tuberosity and linea aspera, 8. greater trochanter, and 9. neck. Another model of the lumbar vertebra is shown in similar fashion in Figure 2 and highlights the following features: 1. body, 2. transverse processes, 3 . inferior articular facet, 4 . superior articular facet, 5 . accessory process, and 6 . spinous process. 

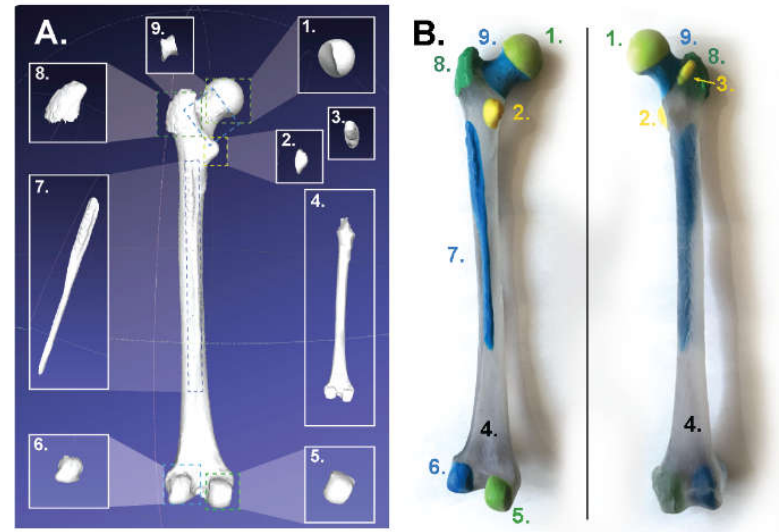

Figure 1. Panel A: Stereolithography (STL) source and split files for the femur (posterior view). Panel B: Poly-jet prints with multiple colors showing posterior and anterior views. Features include 1. head, 2. lesser trochanter, 3 . intertrochanteric line, 4 . body, 5. medial condyle, 6 . lateral condyle, 7 . gluteal tuberosity and linea aspera, 8 . greater trochanter, and 9. neck.
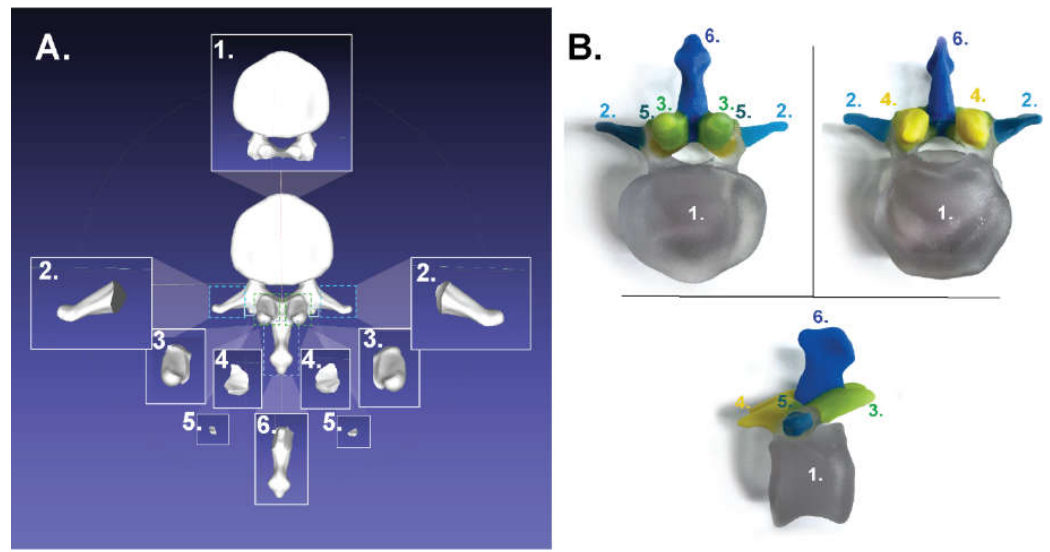

Figure 2. Panel A: STL source and split files for the femur (inferior view). Panel B: Poly-jet prints with multiple colors. Top right shows the superior view, top left shows the inferior view, and bottom shows the sagittal view. Features include 1. body, 2. transverse processes, 3. inferior articular facet, 4 . superior articular facet, 5 . accessory process, and 6 . spinous process.

\subsection{For Binder-Jet Paint Processes}

Four anatomical datasets of a femur, lumbar vertebra, scapula, and innominate bone were chosen to demonstrate multi-color printing on a sandstone binder jet print machine. STL files were imported to Meshmixer and important features colored using the "PaintVertex" tool under "Brushes," again guided by the Pocket Atlas of Human Anatomy. Four views of a colorized femur model at $90^{\circ}$ increments starting with the posterior view are shown in Figure 3. The following features on the femur were highlighted: 1. fovea capitis, 2 . head, 3. greater trochanter, 4 . neck, 5 . lesser trochanter, 6 . trochanteric crest, 7 . spiral line, 8 . gluteal tuberosity, 9 . medial lip of the linea aspera, 10. lateral lip of the linea aspera, 11. medial supracondylar ridge, 12 . medial condyle, 13 . adductor tubercle, 14. medial epicondyle, 15. patellar surface, 16. lateral epicondyle, 17. lateral condyle, 18. intercondylar fossa, 19. lateral supracondylar ridge, and 20. popliteal fossa. The digital model colorized in Meshmixer and re-compiled for display in Meshlab is shown in Figure 3, Panel A. The resultant sandstone full-color printed binder-jet model is shown in Figure 3, Panel B. Superior (left), sagittal (middle), and inferior (right) views of a lumbar vertebra are shown in Figure 4. This model highlights the following anatomical features on a lumbar vertebra: 1 . spinous process, 2 . transverse process, 3 . inferior articular facet, 4 . accessory process, 5. pedicle, 6 . facies intervertebralis, 7 . ring apophysis, and 8 . superior articular facet. The colored 
digital lumbar vertebra colored in Meshmixer and captured in Meshlab is shown in Panel A, while the resultant full-color printed binder-jet model is shown in Panel B. A colorized scapula at superior (top), dorsal (left), and ventral (right) views is shown in Figure 5. The following features of the scapula are highlighted: 1 . superior angle, 2. medial margin, 3. inferior angle, 4. lateral margin, 5 . infraglenoid tubercle, 6. glenoid cavity, 7. neck of scapula, 8. coracoid process, 9. spine of scapula, 10. acromion, 11. acromial articular surface, 12. supraglenoid tubercle, 13. scapular notch, and 14. superior margin. The digital colored digital model is shown in Panel A, and the resultant full-color binder-jet model is shown in Panel B. The colorized innominate bone at superior (left) and inferior (right) views is shown in Figure 6. The following features are highlighted: 1. linea intermedia, 2. tubercle of the iliac crest, 3. external lip of the iliac crest, 4. posterior gluteal line, 5. posterior superior iliac spine, 6. posterior inferior iliac spine, 7. anterior gluteal line, 8. inferior gluteal line, 9. ischial spine, 10. ischial tuberosity, 11. margin of the acetabulum, 12. lunate surface, 13. acetabular fossa, 14. acetabular notch, 15. obturator crest, 16. pubic tubercle, 17. pubic crest, 18. anterior inferior iliac spine, 19. supra-acetabular sulcus, 20. anterior superior iliac spine, 21. internal lip of the iliac crest, 22. pectineal line (continuation of arcuate line) arcuate line, 23. symphyseal surface, 24. arcuate line, 25. auricular surface, 26. iliac tuberosity, 27. posterior obturator tubercle, and 28. anterior obturator tubercle. The digital color model is shown in Panel A, while the resultant full-color printed binder-jet model is shown in Panel B.

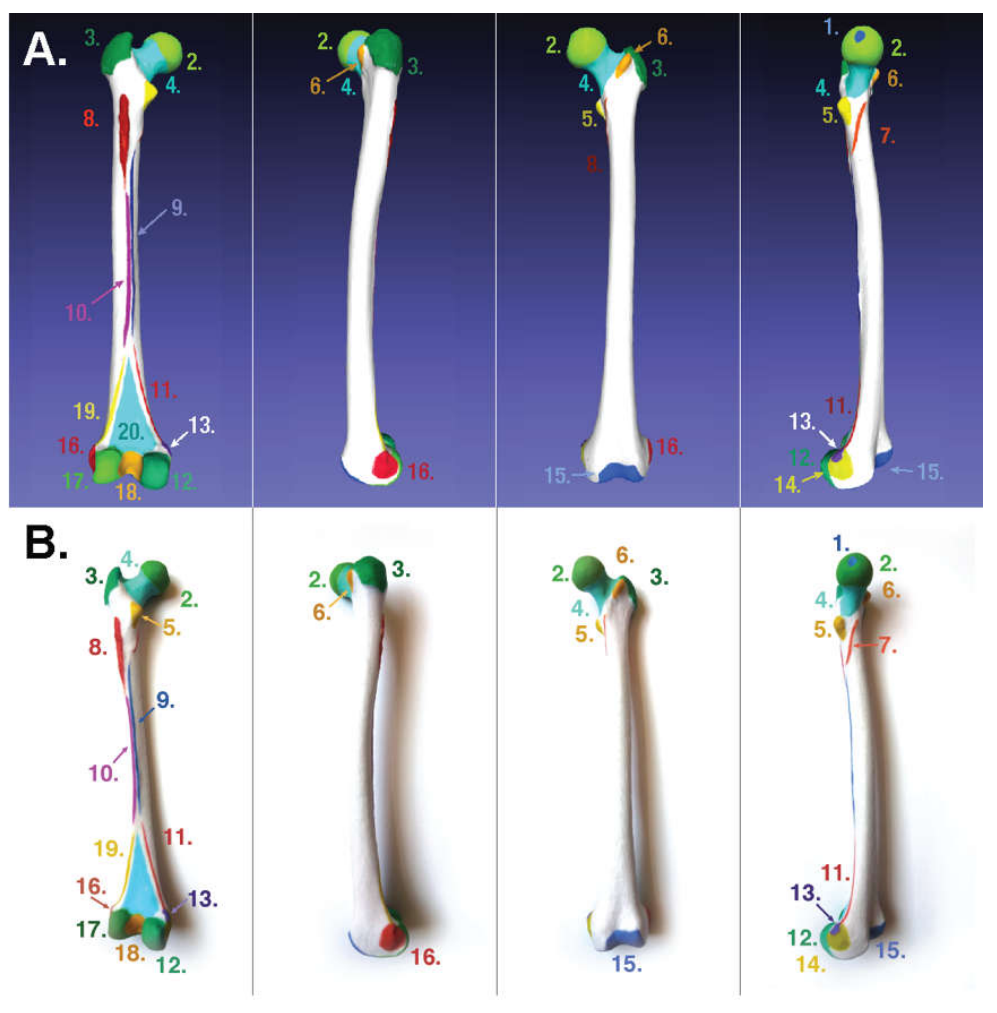

Figure 3. Panel A: Digital colored model (colored in Meshmixer, shown in Meshlab). Panel B: Resulting full-color sandstone binder-jet print of a femur featuring 1. fovea capitis, 2. head, 3. greater trochanter, 4. neck, 5. lesser trochanter, 6. trochanteric crest, 7. spiral line, 8. gluteal tuberosity, 9. medial lip of the linea aspera, 10. lateral lip of the linea aspera, 11. medial supracondylar ridge, 12. medial condyle, 13. adductor tubercle, 14. medial epicondyle, 15. patellar surface, 16. lateral epicondyle, 17. lateral condyle, 18. intercondylar fossa, 19. lateral supracondylar ridge, and 20. popliteal fossa. Image shows four views of the model starting with the dorsal view then rotating $90^{\circ}$ to show all highlighted features. 

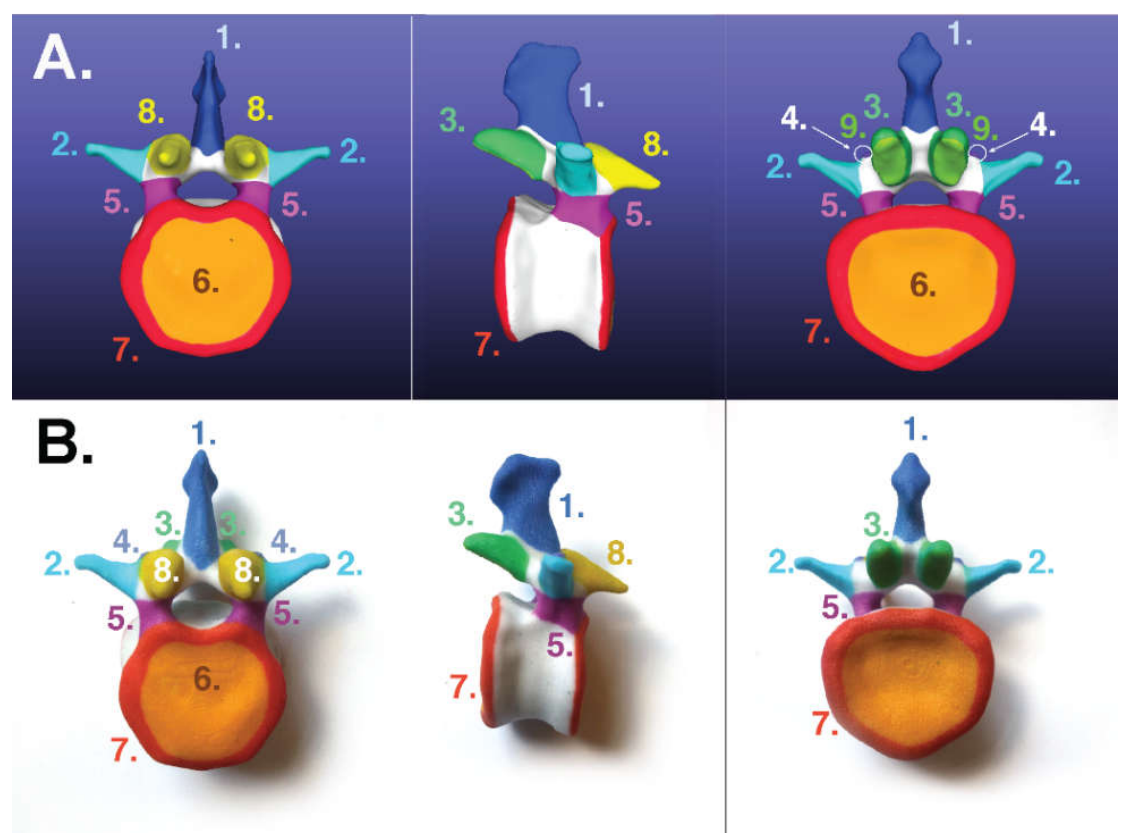

Figure 4. Panel A: Digital colored model (colored in Meshmixer, shown in Meshlab). Panel B: Resulting binder-jet printed lumbar vertebra including 1. spinous process, 2. transverse process, 3 . inferior articular facet, 4. accessory process, 5. pedicle, 6. facies intervertebralis, 7. ring apophysis, and 8. superior articular facet.
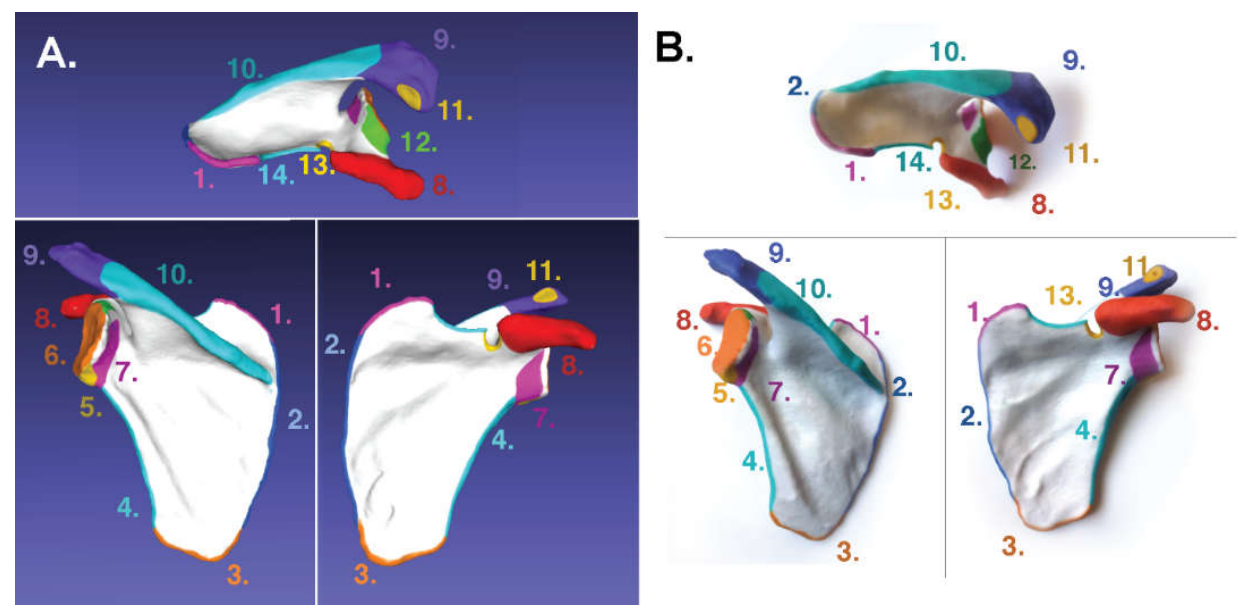

Figure 5. Panel A: Digital colored model. Panel B: resulting binder-jet printed scapula including 1. superior angle, 2. medial margin, 3. inferior angle, 4. lateral margin, 5. infraglenoid tubercle, 6. glenoid cavity, 7. neck of scapula, 8. coracoid process, 9. spine of scapula, 10. acromion, 11. acromial articular surface, 12. supraglenoid tubercle, 13. scapular notch, and 14. superior margin. In each panel, top shows the superior view, left shows the dorsal view, and right shows the ventral view of scapula. 

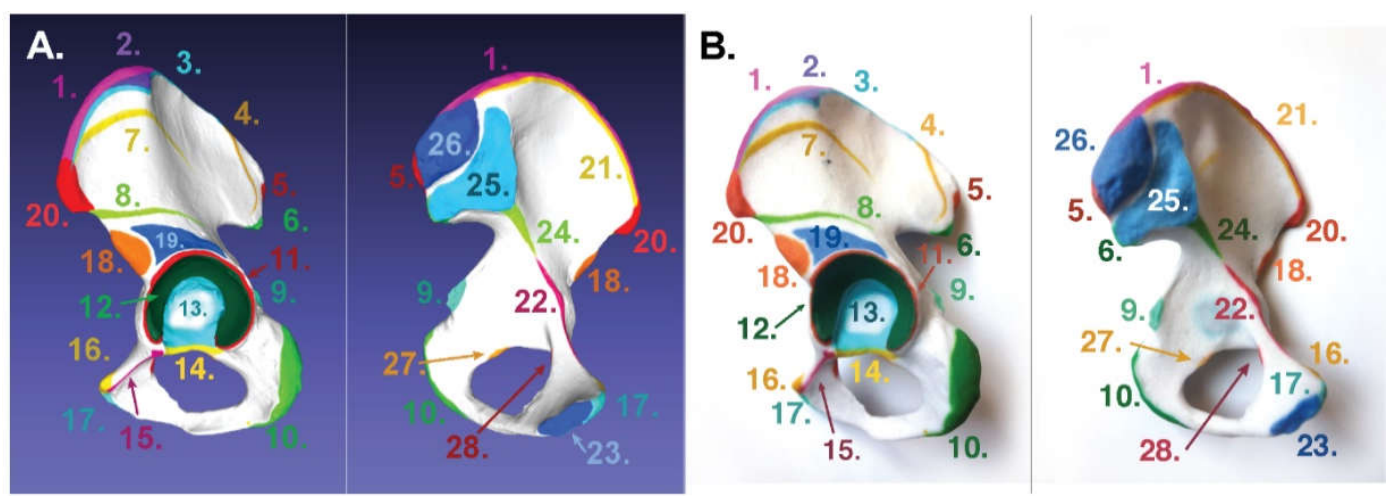

Figure 6. Panel A: Digital colored model. Panel B: Resulting binder-jet printed innominate bone. Features shown include 1. linea intermedia, 2. tubercle of the iliac crest, 3. external lip of the iliac crest, 4. posterior gluteal line, 5. posterior superior iliac spine, 6. posterior inferior iliac spine, 7. anterior gluteal line, 8 . inferior gluteal line, 9 . ischial spine, 10. ischial tuberosity, 11 . margin of the acetabulum, 12. lunate surface, 13. acetabular fossa, 14. acetabular notch, 15. obturator crest, 16. pubic tubercle, 17. pubic crest, 18. anterior inferior iliac spine, 19. supra-acetabular sulcus, 20. anterior superior iliac spine, 21. internal lip of the iliac crest, 22. pectineal line (continuation of arcuate line) arcuate line, 23. symphyseal surface, 24 . arcuate line, 25. auricular surface, 26. iliac Table 27. posterior obturator tubercle, and 28. anterior obturator tubercle.

\section{Discussions}

Three-dimensional surface maps of anatomical data were obtained from the NIH 3D Print Exchange to evaluate two primary strategies for digital color enhancement and 3D printing. The first method utilized a "digital cutting" protocol in which the data were sectioned into anatomical subunits to enable poly-jet printing on a Stratasys Connex3 Objet350. A second strategy utilized "digital painting" to create datasets appropriate for binder-jet printing with a full-color sandstone machine by 3D systems via a third-party company called Shapeways. As noted in Figures 1-6, myriad compelling multi-color anatomical models may be generated via each path.

"Digital cutting" of the model into anatomical subunits was accomplished through the "split complex" function in Meshmixer. This tool allows organic selection of a subregion on the 3D model that is "split" away from the main body. Each anatomical feature to be highlighted was selected in this manner and resulted in multiple files. These were assigned various colors in 3D printing software GrabCad and printed together, which resulted in a single 3D-printed model (Figures 1 and 2).

"Digital painting" of a uniform segmented anatomical dataset is a straightforward approach to instill colors within a model. In this study, the "PaintVertex" tool of the Meshmixer package was used. As the name suggests, each vertex point on the 3D reconstruction model is assigned one specific color and may be accomplished with software packages such as 3DCoat (commercial) and Meshmixer (free). In one example, McMenamin et al. used commercial painting software to create multi-color, sandstone hand models derived from CT scans of cadaveric specimens. In this case, the macro structure of a hand was colored to match tissue anatomy to create a lifelike, compelling model. In the case presented here, freeware was utilized to generate models with a full spectrum of color coding at the substructure level to maximize putative educational utility for learning specific structures.

There are certainly pros and cons associated with the use of digital and poly-jet printing. The painting pathway provides an easier selection of features done in one file using a 10-color palette in Meshmixer's PaintVertex tool. While there is virtually no limit on the number of colors that can be used, distinguishing between similar colors depends on the human eye. Using the available color palette in Meshmixer's PaintVertex tool allows for consistency of color while supplying a variety of hues. We used nine sufficiently distinct colors strategically assigned to spatially distinct features for preparation of VRML files. The same color can be used multiple times as long it is spatially distinct. 
Vertex painting requires less processing power than splitting STLs and can be used to highlight a few dozen features. On the other hand, the poly-jet printing process proved limiting in terms of color and ease of feature selections. The Connex printer used here was a three-headed system, and, while there were at least 45 colors available when using a different color on each head, only five were used for the femur and only six colors were used for the lumbar vertebra since a clear material was also included to better visualize the spatial relationship between subsections. However, clear material is not ideal for color mixing, thus limiting the overall palette on the Connex3 Objet350. Additionally, the use of color "shades" failed (data not shown), as they were visually too close to each other, making it difficult to differentiate among them. Consequently, only six colors were visibly distinct from each other and available for use on our models.

Other practical considerations include durability of printed models, complexity of software processing, pricing, and build area of the 3D printers used. Table 1 details the relative pros and cons of binder-jet printing and poly-jet printing. Firstly, acrylic parts generated with poly-jet technology are widely recognized for better durability compared to sandstone, which is relatively brittle [24]. Secondly, the poly-jet process is limited by hardware and planning. The procedure of splitting the files into multiple parts requires significantly more computer processing power than digitally painting it. Additionally, splitting each model into separate parts requires stepwise planning. For example, the neck and head of the femur must be split from the body before the head can be isolated. The resultant model would comprise multiple files, which can be cumbersome to organize and bring together. Next, poly-jet printing proved to be a lot more expensive. Table A1 (Appendix A) shows that printing via poly-jet is 10-fold that of sandstone binder-jet printing through Shapeways. This price can come down by purchasing an in-house printer; however, this option would require significant initial investment that may be mitigated via access to local fabrication labs. Finally, maximum build size depends on the printer used: $342 \times 342 \times 200 \mathrm{~mm}$ for Connex 3 Objet 350 . The maximum build size for sandstone printing by Shapeways is comparable at $250 \times 380 \times 200 \mathrm{~mm}$.

Table 1. Durability, software processing, pricing, and build size comparisons between poly-jet and binder-jet printing.

\begin{tabular}{ccccc}
\hline & Durability & Software Processing & Pricing & Build Size \\
\hline Poly-jet & High & $\begin{array}{c}\text { Relatively complex (time-consuming, } \\
\text { requires high computational power, } \\
\text { stepwise planning) }\end{array}$ & 10X & $\begin{array}{c}255 \times 252 \times 200 \mathrm{~mm} \\
\text { for Objet 260 Connex3. } \\
342 \times 342 \times 200 \mathrm{~mm} \\
\text { for Objet 350 Connex3 }\end{array}$ \\
\hline Binder-jet & $\begin{array}{c}\text { Relatively } \\
\text { fragile }\end{array}$ & Relatively simple & $\mathrm{X}$ & $250 \times 380 \times 200 \mathrm{~mm}$ \\
\hline
\end{tabular}

Recently, an additional approach combining the file preparation method of binder-jetting and durability of poly-jet printing became available in the Stratasys J750 poly-jet printer. The J750 is a six-head poly-jet printer that allows for printing of 500,000 colors, which was originally announced in 2016 [10]. In July 2019, Shapeways added a software option for full-color poly-jet printing of VRML files on the J750 platform. This announcement is significant for three reasons. Firstly, an increasing prevalence of poly-jet printing technologies enables access via third parties like Shapeways. Secondly, full-color acrylic printing is now practical. Thirdly, VRML files can now be used for poly-jet printing. The capability of J750 in full-color printing will lift the limits associated with Connex machines with fewer than six heads. Additionally, since the J750 accepts VRML files, the process of preparing multi-color digital models is the same as for binder-jet printing. Customers can now upload VRML files, as they did for sandstone printing, and simply select the multi-color poly-jet option. Table A1 (Appendix A) shows prices for the four printed bone models in sandstone and multi-color poly-jet printing through Shapeways, with the latter being over 10 times as expensive. 
Another common method to color a digital model is known as "UV mapping," where a 2D image is projected onto a 3D model. The surface of the 3D model, however, must be "unwrapped" and a 2D image overlaid, thus becoming mapped onto the 3D surface model [25]. This approach is widely used in character modeling, gaming, and 3D animation worlds as it allows for intricate texture while keeping polygon numbers low. It allows for texturing the planes of the mesh, rather than just the vertices. However, UV mapping is not suitable for coloring biomedical data-driven models since they possess a high number of vertices with complex structures that make it especially difficult to "unwrap" and flatten into a 2D plane. Simplifying the model by reducing vertices would come at the cost of anatomical accuracy and should be avoided as much as possible. When 3D-printing colored models with UV mapping, the desired output is a VRML file, which was long associated with sandstone printing. Interactive virtual anatomy models were created with the use of 3D modeling and realistic UV wraps. Fang et al. reported the production of an interactive web platform "Virtual Anatomy System" based on high-resolution anatomical images of the Chinese Visible Human dataset with segmentation through Photoshop and 3D reconstruction through modeling and animation software Maya [26]. Commercial applications such as Complete Anatomy [27] and Visual Anatomy Lite [28] feature realistic 3D models of human anatomy that were UV wrapped. Such models are "digitally modeled" in modeling software such as Maya and 3D Studio Max and wrapped with a painted UV texture [29]. It is important to note that, while these models may heavily reference biomedical data, the 3D surface models are manually created rather than directly utilizing vertex points computed via 3D reconstructions. Our study seeks to introduce a method of producing colorful anatomical models that correlate directly with biomedical data and minimal artistic intervention.

There are several sensible reasons why tangible, physical models derived from biomedical data and enhanced with multi-color landmarks are valuable tools for students. Firstly, an individual no longer needs to project 2D landmarks derived from textbooks onto a 3D mental model. Often, students must put together several 2D illustrations showing multiple views to accurately place the feature on a monochrome, 3D bone specimen. Secondly, the ability to show right and left anatomy, with color enhancement, can reduce confusion. For example, a student need not imagine a mirror model of the 2D illustration for the left femur if both models are present. Thirdly, colored features can also serve as landmarks to which instructors refer for speedy communication in the classroom. Finally, this coloring method defines the edges of each feature, which are not clear using monochrome methods. Students may look to these models for practical study guides.

Ejaz et al. developed color-coded physical heart defect models after separating different colored parts via segmentation. These were used in a premedical human anatomy and physiology class with groups divided into virtual and physical models and subdivided into those with and without colors. The groups were then given heart anatomy identification tests, and the authors concluded that the physical factor was most statistically significant, followed by color [11]. In a second study, Estevez et al. developed color-coded parts for a 3D physical modeling activity of periventricular structures. Students utilizing the colored models scored significantly higher than those taught with traditional 2D methods [30]. It should, however, be noted that this may be attributed to the interactive nature of the physical modeling activity rather than the effective use of color on learning. While these two studies suggest that color-coding 3D physical models promotes learning anatomy, additional research is needed to quantitatively explore their effectiveness.

\section{Conclusions}

This study compared multi-color poly-jet and full-color sandstone printing strategies in the creation of bone models. Both the splitting and the painting approaches utilized freeware to achieve multi-color bone models that highlighted features that first-year anatomy students typically learn. The use of painting was noted as computationally more convenient, while also interfacing with sandstone printers that typically offer more color choices at a fraction of the cost. Nevertheless, acrylic parts maintain advantages in durability, as well as the exclusive use of clear materials that facilitate better 
spatial awareness of related anatomies. In both cases, the combination of colorful visual displays with true biomedical data allows for the production of teaching tools in a cost-effective, straightforward manner. As computing power increases and the cost associated with additive manufacturing falls, full-color models will inevitably become more accessible. Students may soon be able to purchase multi-color bone sets and keep them at home rather than wait for lab access. Moreover, 3D printing also allows for easy manipulation of model sizes. For portability and affordability purposes, students in the future may be able to buy miniaturized versions of bony specimens for independent study. Future studies should probe the effectiveness of multi-color models on student learning by comparing student scores and feedback on multi-color 3D-printed models against traditional 2D illustrations and traditional lab specimens. While biomedical data are used for patient-specific models, their potential use as anatomy teaching tools of specific disease states provides an excellent opportunity for follow-on studies.

Author Contributions: Conceptualization, M.I. and W.M.L.; methodology, M.I. and W.M.L.; validation, M.I. and W.M.L.; formal analysis, M.I. and W.M.L.; investigation, M.I. and T.F.; resources, W.M.L.; data curation (3D printing), A.V.A.; writing. M.I. and T.F.; visualization, M.I.; supervision, W.M.L.; project administration, W.M.L.; funding acquisition, W.M.L. All authors read and agreed to the published version of the manuscript.

Funding: This research received no external funding.

Acknowledgments: The authors graciously thank the Notre Dame Meruelo Family Center for Career Development for the 2019 Summer Funding Program, the Notre Dame Institute of Scholarship for the Liberal Arts for a 2019 Conference Presentation Grant, and the Notre Dame College of Science for a 2019 Travel Grant for Undergraduate Researchers.

Conflicts of Interest: The authors declare no conflicts of interest.

\section{Appendix A}

Table A1. Dimensions and pricing comparisons between full-color sandstone and full-color multi-color poly-jet printing through Shapeways.

\begin{tabular}{ccccc}
\hline & Femur & Lumbar & Scapula & Innominate Bone \\
\hline \multirow{2}{*}{ Dimensions } & $\begin{array}{c}\text { X: } 35.11 \mathrm{~mm} \\
\text { Y: } 30.66 \mathrm{~mm} \\
\text { Z: } 200.83 \mathrm{~mm}\end{array}$ & $\begin{array}{c}\text { X: } 45.16 \mathrm{~mm} \\
\text { Y: } 32.47 \mathrm{~mm}\end{array}$ & $\begin{array}{c}\text { X: } 94.15 \mathrm{~mm} \\
\text { Y: } 113.4 \mathrm{~mm}\end{array}$ & $\begin{array}{c}\text { X: } 61.6 \mathrm{~mm} \\
\text { Y: } 58.76 \mathrm{~mm}\end{array}$ \\
\cline { 2 - 5 } & $\begin{array}{c}\text { Model volume: } \\
34.77 \mathrm{~cm}^{3}\end{array}$ & $\begin{array}{c}\text { Model volume: } \\
13.47 \mathrm{~cm}^{3}\end{array}$ & $\begin{array}{c}\text { Model Volume: } \\
54.06 \mathrm{~cm}^{3}\end{array}$ & $\begin{array}{c}\text { Model volume: } \\
20.88 \mathrm{~cm}^{3}\end{array}$ \\
\hline $\begin{array}{c}\text { Sandstone printing } \\
\text { (full color) }\end{array}$ & $\$ 33.58$ & $\$ 17.60$ & $\$ 48.04$ & $\$ 23.16$ \\
\hline $\begin{array}{c}\text { Multi-color poly-jet } \\
\text { printing }\end{array}$ & $\$ 382.92$ & $\$ 191.19$ & $\$ 556.5$ & $\$ 257.92$ \\
\hline
\end{tabular}

\section{References}

1. Smith, C.F.; Tollemache, N.; Covill, D.; Johnston, M. Take away body parts! An investigation into the use of 3D-printed anatomical models in undergraduate anatomy education. Anat. Sci. Educ. 2018, 11, 44-53. [CrossRef]

2. AbouHashem, Y.; Dayal, M.; Savanah, S.; Strkalj, G. The application of 3D printing in anatomy education. Med. Educ. Online 2018, 20. [CrossRef]

3. Sander, I.M.; Liepert, T.T.; Doney, E.L.; Leevy, W.M.; Liepert, D.R. Patient education for endoscopic sinus surgery: Preliminary experience using 3D-printed clinical imaging data. J. Funct. Biomater. 2017, 8, 13. [CrossRef]

4. Mogali, S.R.; Yeong, W.Y.; Tan, H.K.J.; Tan, G.J.S.; Abrahams, P.H.; Zary, N.; Low-Beer, N.; Ferenczi, M.A. Evaluation by medical students of the educational value of multi-material and multi-colored three dimensional printed models of the upper limb for anatomical education. Anat. Sci. Educ. 2018, 11, 54-64. [CrossRef] 
5. Pawlina, W.; Drake, R.L. Anatomical models: Don't banish them from the anatomy laboratory yet. Anat. Sci. Educ. 2013, 6, 209-210. [CrossRef]

6. Lauridsen, H.; Hansen, K.; Ørum, N.M.; Wang, T.; Pedersen, M. From tissue to silicon to plastic: Threedimensional printing in comparative anatomy and physiology. R. Soc. Open Sci. 2016, 3. [CrossRef] [PubMed]

7. Khot, Z.; Quinlan, K.; Norman, G.R.; Wainman, B. The relative effectiveness of computer-based and traditional resources for education in anatomy. Anat. Sci. Educ. 2013, 6, 211-215. [CrossRef] [PubMed]

8. Preece, D.; Williams, S.B.; Lam, R.; Weller, R. “Let's get physical”: Advantages of a physical model over 3D computer models and textbooks in learning imaging anatomy. Anat. Sci. Educ. 2013, 6, 216-224. [CrossRef] [PubMed]

9. Garas, M.; Vaccarezza, M.; Newland, G.; McVay-Doornbusch, K.; Hasani, J. 3D-printed specimens as a valuable tool in anatomy education: A pilot study. Ann. Anat. 2018, 219, 57-64. [CrossRef] [PubMed]

10. Stratasys. Connex Objet500 and Objet350. Available online: https://www.stratasys.com/3d-printers/objet350-500-connex3 (accessed on 7 January 2020).

11. Ejaz, F.; Ryan, J.; Henriksen, M.; Osborn, M.; Frakes, D. Color-coded patient specific models of congenital heart disease. Rapid Prototyp. J. 2014, 20, 336-343. [CrossRef]

12. Bernhard, J.C.; Isotani, S.; Matsugasumi, T.; Duddalwar, V.; Hung, A.J.; Suer, E.; Baco, E.; Satkunasivam, R.; Djaladat, H.; Metcalfe, C.; et al. Personalized 3D printed model of kidney and tumor anatomy: A useful tool for patient education. World J. Urol. 2016, 34, 337-345. [CrossRef] [PubMed]

13. Yang, D.H.; Kang, J.W.; Kim, N.; Song, J.K.; Lee, J.W.; Lim, T.H. Myocardial 3-dimensional printing for septal myectomy guidance in a patient with obstructive hypertrophic cardiomyopathy. Circulation 2015, 132, 300-301. [CrossRef] [PubMed]

14. Al Jabbari, O.; Abu Saleh, W.K.; Patel, A.P.; Igo, S.R.; Reardon, M.J. Use of three-dimensional models to assist in the resection of malignant tumors. J. Card. Surg. 2016, 31, 581-583. [CrossRef] [PubMed]

15. Costello, J.P.; Olivieri, L.J.; Su, L.; Krieger, A.; Alfares, F.; Thabit, O.; Marshall, M.B.; Yoo, S.J.; Kim, P.C.; Jonas, R.A.; et al. Incorporating three-dimensional printing into a simulation-based congenital heart disease and critical care training curriculum for resident physicians. Congenit. Heart Dis. 2015, 10, 185-190. [CrossRef] [PubMed]

16. Loughborough University. About Additive Manufacturing-Binder Jetting. Available online: https: //www.lboro.ac.uk/research/amrg/about/the7categoriesofadditivemanufacturing/binderjetting/ (accessed on 23 November 2019).

17. 3D Hubs. Full Color Sandstone. Available online: https://www.3dhubs.com/material-group/full-colorsandstone (accessed on 23 November 2019).

18. McMenamin, P.G.; Quayle, M.R.; McHenry, C.R.; Adams, J.W. The production of anatomical teaching resources using three-dimensional (3D) printing technology. Anat. Sci. Educ. 2014, 7, 479-486. [CrossRef]

19. Adams, J.W.; Paxton, L.; Dawes, K.; Burlak, K.; Quayle, M.; McMenamin, P.G. 3D printed reproductions of orbital dissections: A novel mode of visualising anatomy for trainees in ophthalmology or optometry. Br. J. Ophthalmol. 2015, 99, 1162-1167. [CrossRef]

20. Adams, F.; Qiu, T.; Mark, A.; Fritz, B.; Kramer, L.; Schlager, D.; Wetterauer, U.; Miernik, A.; Fischer, P. Soft 3D-printed phantom of the human kidney with collecting system. Ann. Biomed. Eng. 2017, 45, 963-972. [CrossRef]

21. Perica, E.; Sun, Z. Patient-specific three-dimensional printing for pre-surgical planning in hepatocellular carcinoma treatment. Quant. Imaging Med. Surg. 2017, 7, 668-677. [CrossRef]

22. Zheng, J.; Li, C.; Chen, G.; Song, G.; Zhang, Y. Three-Dimensional Printed Skull Base Simulation for Transnasal Endoscopic Surgical Training. World Neurosurg. 2018, 111, 773-782. [CrossRef]

23. Dauber, W.; Feneis, H. Pocket Atlas of Human Anatomy, 5th ed.; Thieme Publishing: Tuebingen, Germany, 2006.

24. Shapeways. Material Information-Sandstone. Available online: https://www.shapeways.com/materials/ sandstone (accessed on 23 November 2019).

25. Blender. UV Editor-Introduction. Available online: https://docs.blender.org/manual/en/latest/editors/uv/ introduction.html (accessed on 23 November 2019).

26. Fang, B.; Wu, Y.; Chu, C.; Li, Y.; Luo, N.; Liu, K.; Tan, L.; Zhang, S. Creation of a Virtual Anatomy System based on Chinese Visible Human data sets. Surg Radiol Anat. 2017, 39, 441-449. [CrossRef] 
27. 3D4Medical. Complete Anatomy 2020. Available online: https://3d4medical.com (accessed on 23 November 2019).

28. Education Mobile. Visual Anatomy. Available online: https://www.edumobapp.com/product.html (accessed on 23 November 2019).

29. Erolin, C. Interactive 3D digital models for anatomy and medical education. Biomed. Vis. 2019, 2, 1-16.

30. Estevez, M.E.; Lindgren, K.A.; Bergethon, P.R. A novel three- dimensional tool for teaching human neuroanatomy. Anat. Sci. Educ. 2010, 3, 309-317. [CrossRef] [PubMed] 